\title{
ON THE EXISTENCE AND NONEXISTENCE OF GLOBAL SOLUTIONS OF REACTION-DIFFUSION EQUATIONS IN SECTORIAL DOMAINS
}

\author{
CATHERINE BANDLE AND HOWARD A. LEVINE
}

\begin{abstract}
In this paper we study the first initial-boundary value problem for $u_{t}=\Delta u+u^{p}$ in conical domains $D=(0, \infty) \times \Omega \subset R^{N}$ where $\Omega \subset S^{N-1}$ is an open connected manifold with boundary. We obtain some extensions of some old results of Fujita, who considered the case $D=R^{N}$.

Let $\lambda=-\gamma_{-}$where $\gamma_{-}$is the negative root of $\gamma(\gamma+N-2)=\omega_{1}$ and where $\omega_{1}$ is the smallest Dirichlet eigenvalue of the Laplace-Beltrami operator on $\Omega$. We prove: If $1<p<1+2 /(2+\lambda)$, there are no nontrivial global solutions. If $1<p<1+2 / \lambda$, there are no stationary solutions in $D-\{0\}$ except $u \equiv 0$. If $1+2 / \lambda<p<(N+1) /(N-3)$ (if $N>3$, arbitrary otherwise) there are singular stationary solutions $u_{s}$. If $u(x, 0) \leq u_{s}(x)$, the solutions are global. If $1+2 / \lambda<p<(N+2) /(N-2)$ and $u(x, 0) \leq u_{s}$, with $u(x, 0) \in C(\bar{D})$, the solutions decay to zero. If $1+2 / N<p$, there are global solutions.

For $1<p<\infty$, there are $L^{\infty}$ data of arbitrarily small norm, decaying exponentially fast at $r=\infty$, for which the solution is not global.

We show that if $D$ is the exterior of a bounded region, there are no global, nontrivial, positive solutions if $1<p<1+2 / N$ and that there are such if $p>1+2 / N$. We obtain some related results for $u_{t}=\Delta u+|x|^{\sigma} u^{p}$ in the cone.
\end{abstract}

\section{INTRODUCTION}

Let $\Omega \subset S^{N-1}$ be a connected submanifold of the unit sphere $S^{N-1}$ in $R^{N}$ with boundary $\partial \Omega \subset S^{N-1}$ and having positive $N-2$ dimensional measure. The boundary is assumed to be smooth enough to permit integration by parts.

By a cone in $R^{N}$ with cross section $\Omega$ with vertex at the origin, we mean the set

$$
D=\{(r, \boldsymbol{\theta}) \mid r>0, \boldsymbol{\theta} \in \Omega\},
$$

where $r=|x|, x \in R^{N}$. The boundary of $D$ is

$$
\partial D=\{(r, \boldsymbol{\theta}) \mid r=0 \text { or } \boldsymbol{\theta} \in \partial \boldsymbol{\Omega}\} .
$$

Received by the editors December 17, 1987 and, in revised form, May 2, 1988.

1980 Mathematics Subject Classification (1985 Revision). Primary 35K20, 35K55.

Key words and phrases. Reaction-diffusion equations, unbounded regions.

The work of Howard A. Levine was supported in part by AFOSR 84-0252, AFOSR 84-0031 and in part by the Schweizerischer Nationalfonds. The U.S. Government is authorized to reproduce and distribute reprints of governmental purposes notwithstanding any copyright notation therein. 
Throughout the remainder of this paper we shall let $\omega_{1}$ denote the smallest Dirichlet eigenvalue for the Laplace-Beltrami operator, namely

$$
\begin{aligned}
\Delta_{\theta} \psi+\omega_{1} \psi=0 & \text { on } \Omega, \\
\psi=0 & \text { on } \partial \Omega .
\end{aligned}
$$

It is known that we may take $\psi>0$ on $\Omega$. We shall assume $\psi$ is normalized so that

$$
\int_{\Omega} \psi(\boldsymbol{\theta}) d S_{\boldsymbol{\theta}}=1
$$

When $N=2, \Omega=(0, \gamma \pi), 0<\gamma<2$ and $\omega_{1}=\gamma^{-2}$ while

$$
\psi=(2 \gamma)^{-1} \sin (\theta / \gamma) .
$$

We wish to consider the long-time behavior of nonnegative solutions of

$$
\begin{aligned}
\partial u / \partial t & =\Delta u+u^{p} & & \text { on } D \times(0, T), \\
u & =0 & & \text { on } \partial D \times(0, T) \text { and at } r=\infty, \\
u(r, \boldsymbol{\theta}, 0) & =u_{0}(r, \boldsymbol{\theta}) & & \text { on } D \times\{0\},
\end{aligned}
$$

where $u_{0} \geq 0$ is given, $p>1$.

We shall use the following terminology: Let

$$
Q_{T}:=D \times(0, T) .
$$

A solution of (1.1) is called quasiregular in $Q_{T}$ if

(i) $u \in C^{2}\left(Q_{T}\right) \cap C^{0}\left(\bar{Q}_{T}-D \times\{T\}\right)$;

(ii) $\forall k>0, \lim _{r \rightarrow \infty} e^{-k r} \int_{\Omega}|u(r, \boldsymbol{\theta}, t)| d S_{\boldsymbol{\theta}}=0$,

$$
\lim _{r \rightarrow \infty} e^{-k r} \int_{\Omega}\left|u_{r}(r, \boldsymbol{\theta}, t)\right| d S_{\boldsymbol{\theta}}=0 .
$$

A quasiregular solution is called regular if there is a sequence $\left\{r_{n}\right\}_{n=1}^{\infty}, r_{n} \rightarrow 0$, such that $\lim _{n \rightarrow \infty} u\left(r_{n}, \boldsymbol{\theta}, t\right) / r_{n}$ exists.

A quasiregular solution is called almost regular if there is a sequence $\left\{r_{n}\right\}_{n=1}^{\infty}$, $r_{n} \rightarrow 0$, such that

$$
\lim _{n \rightarrow \infty} \int_{\Omega}\left[r_{n}^{N-1}\left|u_{r}\left(r_{n}, \boldsymbol{\theta}, t\right)\right|+r_{n}^{N-2}\left|u\left(r_{n}, \boldsymbol{\theta}, t\right)\right|\right] d S_{\boldsymbol{\theta}}=0 .
$$

It is well known [8] that for $u_{0}$ large in the sense that

$$
\frac{1}{p+1} \int_{D} u_{0}^{p+1} d x>\frac{1}{2} \int_{D}\left|\nabla u_{0}\right|^{2} d x
$$

then weak solutions of (1.1) which are (with their gradients) square integrable cannot be global.

Our purpose here, however, is to study (1.1) for "small" initial data.

The plan of the paper is as follows: In $\S 2$ we establish various blowup theorems for small nontrivial initial values. For our major results in this section, we must modify an old argument of Kaplan [6] for bounded domains since 
$\Delta$ has no eigenvalue in the cone. In $\S 3$, we demonstrate the existence of singular stationary solutions for "large $p$," while in $\S 4$ we show that for "small $p>1$ " no regular stationary solutions exist. In $\S 5$, we establish the nonexistence of envelopes of regular stationary solutions. In the next section we show that for certain $p$ no stationary solutions can exist under any singular stationary solutions. In $\S 7$, we demonstrate the existence of global regular solutions of (1.1) which are initially smaller than a singular stationary solution. Finally, in $\S \S 8,9$ we discuss related results for (1.1) in domains exterior to a bounded region and for a generalization of (1.1). In $\S 8$, we show that if $D$ is the exterior of a bounded region, then there are no positive global solutions of (1.1) if $1<p<1+2 / N$, while if $p>1+2 / N$ there are such solutions. (This is the classical result of Fujita [2] when $D=R^{N}$.)

Throughout this paper, when we demonstrate global nonexistence, we shall show that integrals of the form

$$
\int_{D} u(x, t) e^{-k|x|} d x
$$

must become unbounded in a finite time. It is known that if $u$ is smooth on $D \times(0, T)$ and uniformly bounded on $\bar{D} \times[0, T]$, then $u$ is continuable to $D \times[0, T+\delta)$ for some $\delta>0$. However, if such a weighted $L^{1}$ norm (for some $k>0)$ becomes unbounded on $[0, T)$ then $u$ cannot be in $L^{\infty}(D \times[0, T))$. To see this let

$$
M=\sup \{u(x, t) \mid x \in D, t \in[0, T)\}<\infty .
$$

Let

$$
F(t)=\int_{D} u(x, t) e^{-k|x|} d x, \quad t \in[0, T)
$$

Then

$$
F^{\prime}(t) \leq \int_{D} \Delta u e^{-k|x|} d x+M^{p-1} F(t) .
$$

Because of our notion of a regular solution, we may integrate by parts. We obtain

$$
\int_{D} \Delta u e^{-k|x|} d x \leq \int_{D}\left(k^{2}-(N-1) k /|x|\right) u e^{-k|x|} d x \leq k^{2} F(t)
$$

since $N>1$ and $\Delta\left(e^{-k r}\right)=\left(k^{2}-(N-1) k / r\right) e^{-k r}$. We note that since $u \geq 0$, we also have (on $\Omega$ )

$$
\int_{\Omega} \Delta_{\theta} u=\int_{\partial \Omega} \frac{\partial u}{\partial n_{\theta}} \leq 0
$$

Thus

$$
F^{\prime}(t) \leq\left(k^{2}+M^{(p-1)}\right) F(t)
$$

and thus

$$
F(t) \leq F(0) e^{\left(k^{2}+M^{p-1}\right) t} .
$$

Therefore $F$ cannot be unbounded on $[0, T)$ for $T<\infty$. 
Throughout this paper, we shall let $\gamma_{ \pm}$denote the positive and negative roots of $\gamma(\gamma+N-2)=\omega_{1}$, i.e.,

$$
\gamma_{ \pm}=-\frac{1}{2}(N-2) \pm \sqrt{\omega_{1}+\frac{1}{4}(N-2)^{2}} \text {. }
$$

\section{NONEXISTENCE RESULTS FOR $p>1$}

We begin with simple lemma.

Lemma 2.1. Let $m, k, \lambda, \omega$ be real constants with $k^{2}+\lambda>0$. Let $\varphi(r)=$ $r^{m} e^{-k r}$. If one of the conditions

$$
\left(k^{2}+\lambda\right)\left(m^{2}+(N-2) m-\omega\right) \geq\left(m+\frac{1}{2}(N-1)\right)^{2} k^{2}
$$

or

(B)

(i) $\left(k^{2}+\lambda\right)\left(m^{2}+(N-2) m-\omega\right)<\left(m+\frac{1}{2}(N-1)\right)^{2} k^{2}$,

(ii) $m^{2}+(N-2) m-\omega>0$,

(iii) $k\left(m+\frac{1}{2}(N-1)\right)<0$

holds, then

$$
r^{-(N-1)} \frac{d}{d r}\left(r^{N-1} \frac{d \varphi}{d r}\right) \geq \omega r^{-2} \varphi-\lambda \varphi
$$

that is,

$$
\Delta \varphi+\lambda \varphi \geq \omega \varphi r^{-2}
$$

for all $r>0$.

Proof. Direct calculation shows that $(2.1)$ is equivalent to the inequality

$$
\left(k^{2}+\lambda\right) r^{2}-2 k\left(m+\frac{1}{2}(N-1)\right) r+m^{2}+(N-2) m-\omega \geq 0 .
$$

Condition $(A)$ is the statement that the quadratic has no real roots, while condition (B) says that if it does, the larger root is not positive.

We shall need the following simple lemma, which we can trace back to Kaplan [6], who used it to prove global nonexistence.

Lemma 2.2. Let $G(t)$ be a nonnegative $C^{1}$ function defined on $[0, T)$ which satisfies

$$
G^{\prime}(t) \geq(G(t))^{p}-\lambda G(t) \quad \text { for some } \lambda \in \mathbf{R} .
$$

If

(A) $G(0)>0$ and $\lambda \leq 0$, then

$$
T \leq G^{1-p}(0) /(p-1),
$$

while if

(B) $\lambda>0$ and $G(0)>\lambda^{1 /(p-1)}$, then

$$
T \leq \int_{G(0)}^{\infty}\left[\sigma^{p}-\lambda \sigma\right]^{-1} d \sigma:=\tau(\lambda ; G(0)) .
$$


Theorem 2.3. If

$$
1<p<1+2 /\left(N+\gamma_{+}\right)=1+2 /\left(2-\gamma_{-}\right)
$$

then no almost regular solution of (1.1) with nontrivial, nonnegative initial data can exist for all time.

Proof. We must modify Kaplan's method [6] because the Laplacian with Dirichlet boundary conditions has no eigenvalues in cones. Let $u$ be any quasiregular solution of (1.1) and define

$$
\tilde{u}(r, t):=\int_{\Omega} \psi(\boldsymbol{\theta}) u(r, \boldsymbol{\theta}, t) d S_{\boldsymbol{\theta}} \quad \text { and } \quad \tilde{u}_{0}(r)=\tilde{u}(r, 0) .
$$

If we multiply (1.1) by $\psi$ and integrate over $\Omega$ we find, using Jensen's inequality,

$$
\tilde{u}_{t}-\Delta \tilde{u}+\omega_{1} \tilde{u} / r^{2} \geq \tilde{u}^{p} .
$$

For $m>-(N-1)$ put

$$
\Phi_{0}(r)=r^{m} e^{-k r} / C,
$$

where

$$
C=\int_{0}^{\infty} r^{m+N-1} e^{-k r} d r=k^{-(m+N)} \Gamma(m+N) .
$$

From (2.6) we deduce that

$$
\begin{aligned}
\frac{d}{d t} \int_{\varepsilon}^{R} \tilde{u} \Phi_{0} r^{N-1} d r & -\int_{\varepsilon}^{R} \tilde{u}\left(\Delta \Phi_{0}-\left(\omega_{1} / r^{2}\right) \Phi_{0}\right) r^{N-1} d r \\
& +r^{N-1}\left[\tilde{u} \Phi_{0}^{\prime}-\tilde{u}_{r} \Phi_{0}\right]_{\varepsilon}^{R} \geq \int_{\varepsilon}^{R} \tilde{u}^{p} \Phi_{0} r^{N-1} d r .
\end{aligned}
$$

Since $u$ is quasiregular we have

$$
\lim _{R \rightarrow \infty} R^{N-1}\left[\tilde{u} \Phi_{0}^{\prime}-\tilde{u}_{r} \Phi_{0}\right]=0 \text { for any } m \in \mathbf{R} .
$$

Let us assume for the moment that $u$ and $m$ are such that there exists a sequence $\left\{\varepsilon_{n}\right\}_{n=1}^{\infty}, \varepsilon_{n} \rightarrow 0$ with the property

$$
\lim _{n \rightarrow \infty} \varepsilon_{n}^{N-1+m}\left[m \tilde{u}\left(\varepsilon_{n}, t\right) / \varepsilon_{n}-\tilde{u}_{r}\left(\varepsilon_{n}, t\right)\right]=0 .
$$

(This is a consequence of quasiregularity if $m \geq 0$.) Then by (2.7) and Jensen's inequality, the function

$$
G(t):=\int_{0}^{\infty} \tilde{u} \Phi_{0} r^{N-1} d r
$$

satisfies the inequality

$$
G^{\prime}(t) \geq G^{p}(t)+\int_{0}^{\infty} \tilde{u}\left(\Delta \Phi_{0}-\omega_{1} \Phi_{0} / r^{2}\right) r^{N-1} d r .
$$

Next we show that for any initial data $u_{0} \geq 0, u_{0} \not \equiv 0$ there exist $\lambda>0, k$, and $m$ such that

$$
G(0)=C^{-1} \int_{0}^{\infty} r^{m+N-1} e^{-k r} \tilde{u}_{0}(r) d r>\lambda^{1 /(p-1)}
$$


and such that for the corresponding function $G(t)$, the inequality (2.1.2) in Lemma 2.1 holds for $\omega=\omega_{1}$. According to Lemma 2.1(A) we must have

$$
m>\gamma_{+}=-\frac{N-2}{2}+\sqrt{\omega_{1}+\left(\frac{N-2}{2}\right)^{2}}>0
$$

and

$$
\beta:=\frac{\lambda}{k^{2}} \geq\left\{m+\omega_{1}+\frac{(N-1)^{2}}{4}\right\} /\left\{m^{2}+(N-2) m-\omega_{1}\right\} .
$$

In view of condition (2.5), the interval

$$
I=\left(\gamma_{+}, 2 /(p-1)-N\right)
$$

is a nonempty subset of $(0, \infty)$. Hence it is possible to find $m$ such that

$$
\gamma_{+}<m<2 /(p-1)-N .
$$

Once $m$ is fixed, choose $\beta$ such that $(2.10)$ holds. By replacing $C$ by its actual value, (2.9) becomes

$$
k^{-[2 /(p-1)-m-N]} \int_{0}^{\infty} r^{m+N-1} e^{-k r} \tilde{u}_{0} d r>\Gamma(m+N) \beta^{1 /(p-1)} .
$$

By (2.11) we have $2 /(p-1)>m+N$ and we can therefore find a $k$ sufficiently small for which the equality (2.9) is satisfied. With these values of $\beta$ and $k$, we then determine $\lambda$. Consequently, the assumptions of Lemma 2.2(B) hold. Since $m$ is positive, condition (2.8) is certainly satisfied for any almost regular solution. This completes the proof of the theorem.

Remark 2.1. If the initial datum is in $L^{1}(D)$ and if its support contains an open set, then the corresponding almost regular solution cannot be global.

Theorem 2.3 does not exclude the existence of a global solution which is not regular at the origin.

If under the assumptions of the previous theorem a global solution exists, it must be irregular at the vertex of the cone in such a way that (2.8) fails. This observation leads to

Corollary 2.4. If under the assumptions of Theorem 2.3 there exists a quasiregular global solution then

$$
\varliminf_{r \rightarrow 0} r^{\beta} \tilde{u}_{r}(t, r)>0 \quad \text { for all } \beta \in\left(N+\gamma_{+}-1,(3-p) /(p-1)\right)
$$

and for some $t \in(0, \tau(\lambda ; G(0)))$, where $\tau(\lambda ; G(0))$ has been defined in Lemma 2.2 .

If $p>1$ does not satisfy (2.5) we can still find $m$ and $\beta$ satisfying the lefthand side of (2.11) and (2.10), respectively. In this case $2 /(p-1)-m-N<0$ and we cannot always determine $k$ such that $(2.9)$ holds. 
Corollary 2.5. For any $p>1$ and for any $\delta>0$ arbitrarily small, there exist initial data $u_{0}$ with $\left\|u_{0}\right\|_{1}=\delta$ such that the almost regular solution is not global. Proof. Fix $m$ and $\beta$ as above. Observe that $\max r^{m} e^{-k r}=(m / k)^{m} e^{-m}$. Let supp $\tilde{u}_{0} \in(m / k-\varepsilon, m / k+\varepsilon)$ where $\varepsilon>0$ is such that $r^{m} e^{-k r} \geq(m / k)^{m}(2 e)^{-m}$ in this interval. Then

$$
k^{-[2 /(p-1)-m-N]} \int_{0}^{\infty} r^{m+N-1} e^{-k r} \tilde{u}_{0} d r>k^{-[2 /(p-1)-N]} m^{m}(2 e)^{-m}\left\|\tilde{u}_{0}\right\|_{1} .
$$

Hence it is always possible to choose $k$ such that the expression is bounded from below by $\Gamma(m+N) \beta^{1 /(p-1)}$ and, hence, such that (2.9) holds. The assertion is now obtained as Theorem 2.3.

Remark 2.2. If $\Omega=S^{N-1}$ so that $\omega_{1}=0$, the inequality (2.5) becomes

$$
1<p<1+2 / N \text {. }
$$

so that we recover half of Fujita's result [2].

(Observe that

$$
2 /\left(2-\gamma_{-}\right)=4 \cdot\left\{(N+2)+\left[4 \omega_{1}+(N-2)^{2}\right]^{1 / 2}\right\}^{-1} \leq 2 / N
$$

with equality if and only if $\omega_{1}=0$. This says that the range of $p$ 's for which global existence fails for all positive initial data is maximal for the Fujita problem. This is in accordance with the general principle that "small domains are more stable than large domains.") When we set $\omega_{1}=0$, we are passing Dirichlet to "periodic" boundary conditions.

Remark 2.3. When $N=2$, we have $\omega_{1}=\gamma^{-2}$ where $0<\gamma \leq 2$, so that $\omega_{1}(\gamma)$ has range $\left[\frac{1}{4}, \infty\right)$ and the inequality $(2.5)$ becomes

$$
1<p<1+2 \gamma /(2 \gamma+1),
$$

which means that $p$ has maximal range $\left(1, \frac{4}{3}\right)$.

Remark 2.4. Let $\psi_{m}$ be a spherical harmonic of order $m$ and let $\Omega_{m}$ be its nodal domain on $S^{N-1}$. In this case $\omega_{1}=m(N+m-2)$. For the special case

$$
D_{k}=\left\{x: x_{1}>0, \ldots, x_{k}>0\right\}
$$

we have

$$
\psi_{k}=x_{1} \cdot x_{2} \cdots \cdot x_{k} / \sqrt{x_{1}^{2}+x_{2}^{2}+\cdots+x_{k}^{2}}
$$

and hence $\omega_{1}=k(N+k-2)$. In this case the condition for $p$ is $1<p<$ $1+2 /(N+k)$, in accordance with Meier's result [9]. Meier also proved that for $p>1+2 /(N+k)$ both global and nonglobal solutions are possible, a result we also extend here $(\S \S 2,7)$.

When (2.5) fails, i.e., when $p$ exceeds the extreme right member of (2.5), then global solutions may or may not exist. However, when they do, they are not very regular near the vertex of the cone. 
Before we state our next theorem, let us introduce the expressions

$$
\begin{aligned}
& A:=\max \left\{-(N-1),-\omega_{1},-(N-1)^{2} / 4\right\}, \\
& B:=\min \left\{-\frac{1}{2}\left[N-2+\sqrt{4 \omega_{1}+(N-2)^{2}}\right],-(N-1) / 2\right\} .
\end{aligned}
$$

An elementary verification shows that for

$$
\omega_{1} \in \begin{cases}{\left[\frac{1}{4}, 1\right)} & \text { if } N=2, \\ (0, N-1) & \text { if } N>2,\end{cases}
$$

we have $A<B$.

Theorem 2.6. Let $\omega_{1}$ satisfy (2.12). Then the following results hold.

(i) For any $p>1$, no nontrivial global regular solution exists.

(ii) If a global quasiregular solution exists, then

$$
\varliminf_{r \rightarrow 0} r^{N-1+m}\left[m \tilde{u}(r, t)-\tilde{u}_{r}(r, t)\right]>0
$$

for all $m \in(A, B)$ and for some $t \in(0, \tau(0, G(0)))$.

Proof. If (2.1.2) holds for $\lambda=0$, then conditions (B) of Lemma 2.1 must be satisfied; (ii) and (iii) imply that $m<B$ defined above and by (i) we have $m>-\left[\omega_{1}+(N-1)^{2} / 4\right]$. Let $G(t)$ be defined as in the proof of Theorem 2.3. Since (2.8) is supposed to hold for all regular functions, we also require that $m>-N+1$. Thus we have to choose $m$ much that

$$
A<m<B \text {. }
$$

Under this assumption (2.3) holds with $\lambda=0$ and Lemma 2.2(A) applies.

If a global quasiregular solution exists condition (2.8) cannot hold; otherwise the solution could not be global

Remark 2.5. For $N=2$, the condition of Theorem 2.6 becomes $1<\gamma \leq 2$, which means that the sector has a reentrant corner. By Fujita's result, global solutions exist for $p>2$. Consequently, these must be irregular at the corner. The same remark applies when $p>1+2 / N$ and $\Omega$ contains the half-sphere $\left\{x: x_{1}>0,|x|=1\right\}$.

A second global nonexistence theorem may be obtained following the general arguments of Meier [9], which we sketch here.

Let $z\left(t, z_{0}\right)$; solve

$$
\dot{z}=z^{p}, \quad z\left(0, z_{0}\right)=z_{0} .
$$

Let $\delta>0, \lambda, m, k>0$ and define

$$
w(r, \boldsymbol{\theta}, t)=\delta e^{-\lambda t} \varphi(r) \psi(\boldsymbol{\theta}),
$$

where $\varphi$ is as in Lemma 2.1(A). Then we have that

$$
w_{t}-\Delta w \leq 0
$$


in the cone and $w=0$ on the boundary of the cone and at $r=\infty$. One can easily check that

$$
\underline{u}(r, \boldsymbol{\theta}, t)=z(t, w(r, \boldsymbol{\theta}, t))
$$

vanishes on the boundary of the cone, at $r=\infty$, and

$$
\underline{u}_{t}-\Delta \underline{u} \leq \underline{u}^{p}
$$

i.e., $\underline{u}$ is a subsolution.

The subsolution will blow up in some finite time $T$ if

$$
T e^{-(p-1) \lambda T}>\frac{1}{p-1} \max _{D}[w(r, \boldsymbol{\theta}, 0)]^{-(p-1)},
$$

i.e., if

$$
\delta \max _{\boldsymbol{\theta} \in \Omega} \psi(\boldsymbol{\theta})>\left(\frac{\lambda p e}{p-1}\right)^{1 /(p-1)} \cdot\left(\frac{k e}{m}\right)^{m} .
$$

Thus, with $\beta$ given by (2.10), $m$ fixed, and $k$ small where $\lambda \equiv \beta k^{2}$ is also small, (2.17) will hold for fixed $\delta$. Thus, we have

Theorem 2.7. Suppose $m>0$ is such that $\beta$ is given by (2.10) is positive. If there are $\delta>0, k>0$ such that

$$
u_{0}(r, \boldsymbol{\theta}, 0) \geq \delta r^{m} e^{-k r} \psi(\boldsymbol{\theta}),
$$

and such that (2.17) holds with $\lambda=\beta k^{2}$, then $u(r, \boldsymbol{\theta}, t)$ cannot be global.

This result says that for any $p>1$, there exist initial values with $L^{\infty}$ norm as small as one pleases, smaller than $r^{m}$ near $r=0$ for any $m>0$ and which decay exponentially fast at $r=\infty$, for which the corresponding local (in time) solution (when it exists) is not global.

Nonexistence results for more general nonlinearities. Let $f: \mathbf{R}^{+} \rightarrow \mathbf{R}^{+}$be a locally Lipschitz function with the following properties:

(F-0) $f(0)=0, f(s)>0$ for $s>0$;

(F-1) $\lim _{s \rightarrow 0} f(s) / s^{p}=a>0$ for some $p>1$;

(F-2) $\int_{s}^{\infty} 1 / f(\sigma) d \sigma<\infty$ for all $s>0$;

(F-3) $f$ is convex.

Consider the initial value problem (1.1) with $u^{p}$ replaced by $f(u)$. A simple scaling argument shows that we may take $a=1$. the same arguments as in $\S 2.1$ provide nonexistence results for global solutions.

Indeed, multiplying the differential equation by $\psi$ and using Jensen's inequality, we see that with

$$
\tilde{u}(r, t):=\int_{\Omega} \psi u d s_{\theta}
$$

we obtain

$$
\tilde{u}_{t}-\Delta_{r} \tilde{u}+\omega_{1} \tilde{u} / r^{2} \geq f(\tilde{u}) \quad \text { in }(0, \infty) \times(0, T) .
$$


Note that this is a simple extension of (2.6). If $u$ is regular solution, then we deduce, as in the proof of Theorem 2.3, that the corresponding function $G(t)$ satisfies

$$
G^{\prime}(t) \geq f(G)-\lambda G
$$

An immediate generalization of Lemma 2.2 is

Lemma 2.8. For $\lambda>0$ denote the positive solution of $f(s)-\lambda s=0$ by $s_{\lambda}$. if $G(0)>s_{\lambda}$ then $G(t)$ cannot exist beyond

$$
\tau_{\lambda}=\int_{G(0)}^{\infty} \frac{d s}{f(s)-\lambda s} .
$$

From $\left(2.3_{f}\right)$ and this lemma we obtain the following generalizations of Theorems 2.3 and 2.6 .

Theorem 2.9. Assume (F-0)-(F-3) hold.

(i) If (2.5) holds, then the same statement as in Theorem 2.3 holds for the solutions of (1.1) with $u^{p}$ replaced by $f(u)$.

(ii) The statement of Theorem 2.6 remains valid for the solutions of (1.1) with $u^{p}$ replaced by $f(u)$.

Proof. Obviously it suffices to prove the theorem for small initial values. By (F-1) we have

$$
s_{\lambda} \sim(\lambda / a)^{1 /(p-1)} \text { as } \lambda \rightarrow 0 .
$$

Therefore there exists a constant $\lambda_{0}>0$ such that

$$
s_{\lambda}<(2 \lambda / a)^{1 /(p-1)} \text { for } 0<\lambda \leq \lambda_{0} .
$$

We can now proceed exactly as in the proof of Theorem 2.3.

First fix $m \in I$. Then determine $k_{0}>0$ such that

$$
\left.G(0)>\left(2 \beta k^{2}\right) / a\right)^{1 /(p-1)} \text { for all } 0<k \leq k_{0} .
$$

By (2.19) we have

$$
G(0) \geq s_{\beta k^{2}} \quad \text { for } k \leq \min \left\{k_{0}, \sqrt{\lambda_{0} / \beta}\right\}=: k^{\prime} .
$$

If $\lambda>\beta k^{\prime 2}$, then $G(0)>s_{\lambda}$.

The first assertion is now obvious. The remainder of the proof is a repetition of the proof of Theorem 2.5 and is omitted.

Remark 2.6. The results hold for every nonlinearity $\hat{f}(s) \geq f(s)$, where $f$ is as above. This follows immediately from the standard comparison principle for the corresponding solutions $\hat{u}$ and $u$, i.e., $\hat{u}(x, t) \geq u(x, t)$.

\section{THE EXISTENCE OF SINGULAR SOLUTIONS}

Under certain conditions, we may construct singular stationary solutions. For example $N=2$ and

$$
p>2 \gamma+1,
$$


let $\alpha_{M}$ be the unique root of

$$
\frac{1}{2} \gamma \pi=\int_{0}^{1}\left[\psi\left(p, \alpha_{M}, \theta\right)\right]^{-1 / 2} d \theta
$$

where

$$
\psi\left(p, \alpha_{M}, \theta\right) \equiv(2 /(p-1))^{2}\left(1-\theta^{2}\right)+\left[2 \alpha_{M}^{p-1} /(p-1)\right]\left(1-\theta^{p+1}\right) .
$$

Define $\alpha(\theta)$ by

$$
\theta=\int_{0}^{\alpha(\theta) / \alpha_{M}}\left(\psi\left(p, \alpha_{M}, \theta\right)\right)^{1 / 2} d \theta
$$

for $\theta \in\left[0, \frac{1}{2} \gamma \pi\right]$ and by

$$
\alpha(\theta)=\alpha(\gamma \pi-\theta)
$$

for $\theta \in\left[\frac{1}{2} \gamma \pi, \gamma \pi\right]$. Then

$$
u_{s}(r, \theta)=r^{-2 /(p-1)} \alpha(\theta), \quad r>0,0 \leq \theta \leq \gamma \pi,
$$

is a solution of the stationary problem for (1.1) except at $r=0$.

In higher dimensions, such singular solutions will exist under circumstances analogous to the condition $p>1+2 \gamma$.

We consider

$$
\Delta u+u^{p}=0 \text { in } D, \quad u=0 \text { in } \partial D,
$$

and we look for a solution of the type

$$
u(r, \boldsymbol{\theta})=r^{q} \alpha(\boldsymbol{\theta}) .
$$

Since

$$
\Delta=\frac{\partial^{2}}{\partial r^{2}}+\frac{N-1}{r} \frac{\partial}{\partial r}+\frac{1}{r^{2}} \Delta_{\theta},
$$

we find with this choice that

$$
r^{q-2} \Delta_{\theta} \alpha+q(N+q-2) r^{q-2} \alpha+r^{p q} \alpha^{p}=0 .
$$

Setting $q=-2 /(p-1)$, the equations for $\alpha$ become

$$
\Delta_{\boldsymbol{\theta}} \alpha+\nu \alpha+\alpha^{p}=0 \quad \text { in } \Omega, \quad \alpha=0 \quad \text { on } \partial \Omega,
$$

where

$$
\nu=\frac{2}{p-1}\left(\frac{2}{p-1}+2-N\right) .
$$

By standard arguments we have

Lemma 3.1. For any $p>1$ with

$$
p< \begin{cases}(N+1) /(N-3) & \text { if } N \geq 4, \\ \infty & \text { if } N=2,3,\end{cases}
$$


problem (3.3) possesses a nontrivial positive solution provided that $\nu<\omega_{1}$. If $\omega_{1} \leq \nu$, no such solution of (3.3) exists.

Proof. We shall only sketch the main ideas and refer to the standard literature for more details. The second statement follows if we multiply (3.3) by $\psi(\boldsymbol{\theta})$ and integrate over $\Omega$.

For any $v \in W_{0}^{1,2}(\Omega) \cap L^{p+1}(\Omega)$ define

$$
J[v]:=\frac{\mathbf{D}_{s}(v)-\nu \int_{\Omega} v^{2} d S_{\boldsymbol{\theta}}}{\left(a \int_{\Omega} v^{p+1} d S_{\boldsymbol{\theta}}\right)^{2 / p+1}}
$$

where $\mathbf{D}_{s}(v)$ stands for the Dirichlet integral over $\Omega \subset S^{N-1}$ and $d S_{\boldsymbol{\theta}}$ is the surface element on $S$.

By our assumption we have

$$
\mathbf{D}_{s}(v) \geq \omega_{1} \int_{\Omega} v^{2} d S_{\boldsymbol{\theta}}>\nu \int_{\Omega} v^{2} d S_{\boldsymbol{\theta}}
$$

for all $v \neq 0$. Hence $J[v]$ is bounded from below.

Let $\left\{v_{n}\right\}_{n=1}^{\infty}$ be a minimizing sequence such that

$$
\left\|v_{n}\right\|_{L^{p+1}(\Omega)}=1 \quad \text { and } \quad \lim _{n \rightarrow \infty} J\left[v_{m}\right]=\inf _{v} J[v] .
$$

Obviously $\left\{v_{n}\right\}_{n=1}^{\infty}$ is bounded in $W_{0}^{1,2}(\Omega)$. Therefore there exists a subsequence, say $\left\{v_{n}\right\}_{n=1}^{\infty}$, such that

$$
v_{n} \rightarrow v \quad \text { weakly in } W_{0}^{1,2}(\Omega)
$$

and

$$
v_{n} \rightarrow v \text { strongly in } L^{p+1}(\Omega) .
$$

For the next step, we show that $v \in W_{0}^{1,2}(\Omega) \cap L^{p+1}(\Omega)$ satisfies the Euler equation

$$
\Delta_{\boldsymbol{\theta}} v+\nu v+\lambda v^{p}=0 \text { in } \Omega
$$

for some appropriate value of $\lambda>0$. The function $\alpha(\boldsymbol{\theta})=\lambda^{1 /(p-1)} v(\boldsymbol{\theta})$ has the required properties.

From these considerations, we have

Theorem 3.1. Suppose $\omega_{1}>\nu$ and

$$
1<p< \begin{cases}(N+1) /(N-3) & \text { if } N>3, \\ \infty & \text { if } N=2,3 .\end{cases}
$$

That is,

$$
1-2 / \gamma_{-}<p< \begin{cases}(N+1) /(N-3) & \text { if } N>3 \\ \infty & \text { if } N=2,3 .\end{cases}
$$

There exists a singular solution to (3.1) of the form

$$
u_{s}(r, \boldsymbol{\theta})=r^{-2 /(p-1)} \alpha(\boldsymbol{\theta}),
$$


where $\alpha$ is a solution to (3.3). No positive singular solution of the above form exists if $\omega_{1} \leq \nu$.

Remark 3.1. Let $D_{k}$ be as in Remark 2.4 of $\S 2$. Then $u_{s}$ exists whenever

$$
\frac{2}{p-1}\left(\frac{2}{p-1}+2-N\right)<k(N+k-2) \text {. }
$$

Remark 3.2. Notice that if $U(\mathbf{x})$ solves $(3.1)$, so does $V(\mathbf{x} ; \lambda)=\lambda^{2 / p-1} U(\lambda \mathbf{x})$ for any $\lambda>0$.

Lemma 3.2. The only solutions of (3.1) such that for all $\lambda>0, \lambda^{2 / p-1} u_{s}(\lambda \mathbf{x})=$ $u_{s}(\mathbf{x})$ are of the form (3.6).

Proof. Let $V(\mathbf{x} ; \lambda)$ be any such solution. Then differentiation with respect to $\lambda$ yields

$$
0=(2 /(p-1)) \lambda^{-1} V+r V_{r}
$$

and in particular for $\lambda=1$

$$
0=(2(p-1)) V+r V_{r} .
$$

Integration of this first-order equation yields the desired result.

\section{NONEXISTENCE OF STATIONARY SOLUTIONS FOR SMALL $p$}

Again let

$$
\gamma_{ \pm}=\frac{1}{2}\left[-(N-2) \pm \sqrt{(N-2)^{2}+4 \omega_{1}}\right] .
$$

In this section, we show that if

$$
p<1-2 / \gamma_{-}
$$

then there are no stationary solutions of (1.1), i.e., no $w>0$ such that

$$
\Delta w+w^{p}=0 \quad \text { in } D, \quad w=0 \quad \text { on } \partial D .
$$

When $N=2,(4.0)$ reduces to $p<2 \gamma+1$. The argument proceeds via a series of lemmas. Let $u$ solve $\Delta u+u^{p}=0$ in $D, u \in C^{0}(\bar{D}) \cap C^{2}\left(D^{\prime}\right)$ for all $D^{\prime} \subset D$, $D^{\prime}$ bounded, and let $u=0$ on $\partial D$. Define

$$
\tilde{u}(r)=\int_{\Omega} \psi(\boldsymbol{\theta}) u(r, \boldsymbol{\theta}) d S_{\boldsymbol{\theta}} .
$$

Then

$$
\begin{aligned}
\Delta \tilde{u} & =\Delta_{r} \tilde{u}=r^{-(N-1)} \frac{\partial}{\partial r}\left(r^{N-1} \frac{\partial \tilde{u}}{\partial r}\right)=\int_{\Omega} \psi(\theta) \Delta_{r} u(r, \theta) d \theta \\
& =\int_{\Omega} \psi\left[-\frac{1}{r^{2}} \Delta_{\theta} u-u^{p}\right] d \theta .
\end{aligned}
$$

Hence, by Jensen's inequality,

$$
\Delta \tilde{u}-\left(\omega_{1} / r^{2}\right) \tilde{u}+\tilde{u}^{p} \leq 0
$$


for $r>0$. We see also that

$$
\left(\Delta_{r}-\omega_{1} / r^{2}\right) \tilde{u}=r^{-(\beta-\lambda)}\left(r^{\beta}\left(r^{-\lambda} \tilde{u}\right)^{\prime}\right)^{\prime}
$$

where $\lambda$ is one of $\gamma_{+}, \gamma_{-}$and $\beta=N-1+2 \lambda$, so that we have instead of (4.2),

$$
\left(r^{\beta}\left(r^{-\lambda} \tilde{u}\right)^{\prime}\right)^{\prime}+r^{\beta-\lambda} \tilde{u}^{p} \leq 0
$$

Let

$$
l(r)=r^{-\lambda} \tilde{u}(r)
$$

for the sake of convenience. Then, for $0<\rho<r<\infty$,

$$
r^{\beta} l^{\prime}(r)-\rho^{\beta} l^{\prime}(\rho)+\int_{\rho}^{r} s^{\beta-\lambda} \tilde{u}^{p}(s) d s \leq 0
$$

and, after a second quadrature,

$$
l\left(r_{1}\right)-l\left(r_{2}\right)+\int_{r_{2}}^{r_{1}} \frac{d \xi}{\xi^{\beta}}\left(\int_{\rho}^{\xi} s^{\beta-\lambda} \tilde{u}^{p}(s) d s\right) d \xi \leq \frac{r_{2}^{1-\beta}-r_{1}^{1-\beta}}{1-\beta} \rho^{\beta} l^{\prime}(\rho)
$$

provided $\beta \neq 1$ and $\rho<r_{2}<r_{1}$.

We are now in a position to prove some lemmas.

Lemma 4.1. Let $\lambda=\gamma_{+}$. Then

$$
\lim _{\rho \rightarrow 0} \rho^{\beta} l^{\prime}(\rho)=0 .
$$

Proof. The function $h(r, \boldsymbol{\theta})=r^{\lambda} \psi(\boldsymbol{\theta})$ is harmonic. Therefore, if we set

$$
D_{\varepsilon}=\{(r, \boldsymbol{\theta}) \mid \varepsilon<r<1, \boldsymbol{\theta} \in \Omega\},
$$

we find that

$$
0=\int_{D_{\varepsilon}} h \Delta u d x+\int_{D_{\varepsilon}} h u^{p} d x=\oint_{\partial D_{\varepsilon}}\left[h \frac{\partial u}{\partial n}-u \frac{\partial h}{\partial n}\right] d s+\int_{D_{\varepsilon}} h u^{p} d x .
$$

Let $\Gamma_{a}=\partial D_{\varepsilon} \cap\{r=a\}$. Then, we have

$$
\begin{aligned}
0= & -\varepsilon^{\lambda+N-1} \int_{\Omega} \psi(\boldsymbol{\theta}) u_{r}(\varepsilon, \boldsymbol{\theta}) d S_{\theta}+\lambda \varepsilon^{\lambda+N-2} \int_{\Omega} \psi(\boldsymbol{\theta}) u(\varepsilon, \boldsymbol{\theta}) d S_{\boldsymbol{\theta}} \\
& +\int_{\Gamma_{1}}\left(h \frac{\partial u}{\partial r}-u \frac{\partial h}{\partial r}\right) d s+\int_{D_{\varepsilon}} h u^{p} d x,
\end{aligned}
$$

whence

$$
\varepsilon^{\lambda+N-1}\left[\tilde{u}^{\prime}(\varepsilon)-\lambda \tilde{u}(\varepsilon) / \varepsilon\right]=\int_{D_{\varepsilon}} h u^{p} d x+C,
$$

where $C$ is a constant independent of $\varepsilon$. The left-hand side of this may be rewritten in terms of $\beta$, i.e.,

$$
\varepsilon^{\beta} l^{\prime}(\varepsilon)=\int_{D_{\varepsilon}} h u^{p} d x+C .
$$


Since $C$ does not depend on $\varepsilon$ and the integral is a decreasing function of $\varepsilon$, $\lim _{\varepsilon \rightarrow 0} \varepsilon^{\beta} l^{\prime}(\varepsilon)$ exists. Suppose this limit is $\kappa \neq 0$. Then for all $\delta \in(0,|\kappa|)$ there is $\rho_{0}$ such that

$$
(\kappa-\delta) / \rho^{\beta} \leq l^{\prime}(\rho) \leq(\kappa+\delta) / \rho^{\beta}
$$

if $\rho \leq \rho_{0}$. Integrating this over $\left[\varepsilon, \rho_{0}\right]$, we find

$$
\frac{\kappa-\delta}{\beta-1}\left[\varepsilon^{1-\beta}-\rho_{0}^{1-\beta}\right] \leq l\left(\rho_{0}\right)-l(\varepsilon) \leq \frac{\kappa+\delta}{\beta-1}\left[\varepsilon^{1-\beta}-\rho_{0}^{1-\beta}\right] .
$$

Now for $\lambda=\frac{1}{2}(\beta-N+1)$,

$$
\lim _{r \rightarrow 0} r^{\lambda} l(r)=\tilde{u}(0)=0 .
$$

If we multiply (4.6) through by $\varepsilon^{\lambda}$, we have

$$
\frac{\kappa-\delta}{\beta-1}\left[\varepsilon^{2-N-\lambda}-\varepsilon^{\lambda} \rho_{0}^{1-\beta}\right] \leq \varepsilon^{\lambda} l\left(\rho_{0}\right)-\varepsilon^{\lambda} l(\varepsilon) \leq \frac{\kappa+\delta}{\beta-1}\left[\varepsilon^{2-N-\lambda}-\varepsilon^{\lambda} \rho_{0}^{1-\beta}\right] .
$$

Since $2-N-\lambda<0$, we have a contradiction if $\varepsilon \rightarrow 0^{+}$unless $\kappa=0$.

Thus, from 4.4 , we see that

$$
r^{\beta} l^{\prime}(r)+\int_{0}^{r} s^{N+\lambda-1} \tilde{u}^{p}(s) d s \leq 0 .
$$

Lemma 4.2. Let $\lambda=\gamma_{+}$. Then $l(r)$ is decreasing and

$$
\lim _{r \rightarrow \infty} l(r)=0 \text {. }
$$

Proof. The first statement follows from (4.7). Let $l_{0}=\lim _{r \rightarrow \infty} l(r)$. Assume $l_{0} \neq 0$. Then from (4.7) we have

$$
l(r)-l(\rho)+\int_{\rho}^{r} \xi^{-\beta} d \xi \int_{0}^{\xi} s^{[N-1+\lambda(p+1)]} l^{p}(s) d s \leq 0 .
$$

Thus

$$
\int_{\rho}^{\infty} \xi^{-\beta} d \xi \int_{0}^{\xi} s^{[N-1+\lambda(p+1)]} l^{p}(s) d s<\infty .
$$

Therefore, since $l^{\prime}(r) \leq 0$, we have

$$
l_{0}^{p} \int_{\rho}^{\infty} \xi^{[1+(p-1) \lambda]} d \xi<\infty,
$$

which is not possible. Thus, $l_{0}=0$.

Lemma 4.3. Let $\lambda=\gamma_{+}$. Then

$$
\lim _{r \rightarrow \infty} r^{(2+(p-1) \lambda) / p} l(r)=0 .
$$

Proof. From Lemma 4.2 and (4.8), we see that

$$
\lim _{r \rightarrow \infty} \int_{r}^{2 r} \xi^{-\beta} d \xi \int_{0}^{\xi} s^{[N-1+\lambda(p+1)]} l^{p}(s) d s=0 .
$$


By the monotonicity of $l(\cdot)$,

$$
\begin{aligned}
& l^{p}(2 r) \int_{r}^{2 r} \xi^{-\beta} d \xi \int_{0}^{\xi} s^{[(N-1)+\lambda(p+1)]} d s \\
& \quad \leq \int_{r}^{2 r} \xi^{-\beta} d \xi \int_{0}^{\xi} s^{[N-1+\lambda(p+1)]} l^{p}(s) d s
\end{aligned}
$$

so that

$$
\lim _{r \rightarrow \infty} l^{p}(2 r)\left\{(2 r)^{[2+\lambda(p-1)]}\left[1-\left(\frac{1}{2}\right)^{2+\lambda(p-1)}\right]\right\}=0
$$

and we have the result.

Thus, we obtain

Corollary 4.4. Let $\lambda=\gamma_{+}$; then

$$
\lim _{r \rightarrow \infty} r^{\prime \prime} l(r)=0
$$

for all

$$
\nu<(2+(p-1) \lambda) /(p-1) .
$$

Proof. Let $a=2+(p-1) \lambda$. In (4.8) take $\rho=r / 2$. Then, since $l(r) \leq l(\eta)$ for $\eta \in(0, r]$ and some $c>0$,

$$
\begin{aligned}
-l(r)+l(r / 2) & \geq \int_{r / 2}^{r} \xi^{-\beta} d \xi \int_{0}^{\xi} s^{N-1+(p+1) \lambda} l^{p}(s) d s \\
& \geq c l^{p}(r) r^{a},
\end{aligned}
$$

we see that

$$
\lim _{r \rightarrow \infty} r^{(a / p+a)} l^{p}(r)=0 .
$$

Therefore

$$
\lim _{r \rightarrow \infty} r^{\left(a / p+a / p^{2}\right)} l(r)=0 .
$$

Repeating this argument yields

$$
\lim _{r \rightarrow \infty} r^{a\left(1 / p+1 / p^{2}+\cdots\right)} l(r)=0 .
$$

Lemma 4.5. Let $\lambda=\gamma_{+}$. There exists $c>0$ such that

$$
l(r) r^{N-2+2 \lambda} \geq c \quad \text { if } r \geq 1 .
$$

Proof. From (4.7), for $r \geq 1$,

$$
r^{\beta} l^{\prime}(r)+\int_{0}^{1} s^{N-1+\lambda} \tilde{u}^{p}(s) d s \leq 0
$$

so that, with $c_{0}$ as the integral on the left,

$$
l(r)-l(\rho)+\left(c_{0} /(\beta-1)\right)\left[\rho^{1-\beta}-r^{1-\beta}\right] \leq 0 .
$$

Letting $r \rightarrow \infty$, we have

$$
l(\rho) \geq c \rho^{1-\beta} \quad(\rho \geq 1) .
$$


That is, for $\rho \geq 1$,

$$
l(\rho) \cdot \rho^{N-2+2 \lambda} \geq c_{1},
$$

whence the statement of the lemma.

From Corollary 4.4 and Lemma 4.5 we must have (with $\lambda=\gamma_{+}$)

$$
(2-\lambda+p \lambda) /(p-1) \leq N-2+2 \lambda
$$

or

$$
p \geq 1+2 /(N-2+\lambda) .
$$

We thus obtain the following nonexistence result.

Theorem 4.6. If

$$
p<(2+\lambda) / \lambda \quad\left(\lambda=-\gamma_{-}\right),
$$

then no positive stationary solution exists.

Theorem 4.7. If a stationary solution exists then $\tilde{u}(r) r^{\nu} \rightarrow 0$ as $r \rightarrow 0$ for all $\nu<2 /(p-1)$.

Remark 4.1. If $\lambda=-\gamma_{-}$and

$$
\frac{2+\lambda}{\lambda}<p< \begin{cases}(N+1) /(N-3), & N>3, \\ \infty, & N=2,3,\end{cases}
$$

then there exist singular solutions, as we recall from the preceding section (equation 3.5).

Remark 4.2. Similar arguments show that if

$$
p< \begin{cases}N /(N-2), & N>2, \\ \infty, & N=2,\end{cases}
$$

then (4.1) has no nontrivial positive global solutions if $D=R^{N}$. See $\S 9$ and [14]. (In the case $D=R^{N}$ such ground states actually exist if and only if $p>(N+2) /(N-2)$. See [22].

\section{ON ENVELOPES}

We turn again to (4.1). Suppose $w>0$ is a regular stationary solution of (4.1). Then it is easy to verify that

$$
v(r, \theta ; \lambda)=\lambda^{2 /(p-1)} w(\lambda r, \theta)
$$

is also such a stationary solution. The envelope of such solutions, when it exists, is given by

$$
V(r, \boldsymbol{\theta})=v(r, \boldsymbol{\theta}, \lambda(r, \boldsymbol{\theta}))
$$

where $\lambda$ is found from

$$
v_{\lambda}(r, \boldsymbol{\theta}, \lambda(r, \boldsymbol{\theta}))=0 .
$$


One easily checks that if $\lambda_{r}, \nabla_{\theta} \lambda$ exist, then $V$ is also a stationary solution of the differential equation in (4.1). Writing out (5.3) we see that with

$$
\rho \equiv r \lambda(r, \boldsymbol{\theta}),
$$

$\lambda(r, \theta)$ exists if and only if $\rho$ solves

$$
G(\rho, \boldsymbol{\theta})=\rho w_{\rho}(\rho, \boldsymbol{\theta})+(2 /(p-1)) w(\rho, \boldsymbol{\theta}) \equiv 0,
$$

as a function of $\boldsymbol{\theta}$ in a $C^{1}$ fashion on $\Omega$.

Leamma 5.1. There is no regular, positive stationary solution of (1.1) for which the solution set of $(5.4)$ contains a $C^{1}$ surface $\rho=\rho(\theta)$ defined on a nonempty open subset $\Omega_{0} \subset \Omega$.

Proof. If, to the contrary, such a surface existed on some open $\Omega_{0} \subset \Omega, \Omega_{0} \neq$ $\varnothing$, let $\lambda(r, \boldsymbol{\theta})=\rho(\boldsymbol{\theta}) / r$ and set

$$
\begin{aligned}
V(r, \boldsymbol{\theta}) & =(\rho(\boldsymbol{\theta}))^{2 /(p-1)} w(\rho(\boldsymbol{\theta}), \boldsymbol{\theta}) r^{-2 /(p-1)} \\
& =F(\boldsymbol{\theta}) r^{-2 /(p-1)}=v(r, \boldsymbol{\theta}, \lambda(r, \boldsymbol{\theta})) .
\end{aligned}
$$

Since $\rho(\theta)$ is $C^{1}$, so is $\lambda$ and hence $V$ solves $\Delta V+V^{p}=0$ in the subcone $(0, \infty) \times \Omega_{0}$. Consequently, $F(\boldsymbol{\theta})$ must be a positive solution of the differential equation (3.3) with $\nu$ given prior to Lemma 3.1. (We do not require $F$ to vanish on $\partial \Omega_{0}$.) We have $w(\rho(\boldsymbol{\theta}), \boldsymbol{\theta})=V(\rho(\boldsymbol{\theta}), \boldsymbol{\theta})$ in $\Omega_{0}$, while, in view of (5.4) and the chain rule,

$$
\nabla_{\boldsymbol{\theta}} F=\rho^{2 /(p-1)}(\boldsymbol{\theta}) \nabla_{\boldsymbol{\theta}} w(\rho(\boldsymbol{\theta}), \boldsymbol{\theta}),
$$

i.e.,

$$
\nabla_{\boldsymbol{\theta}} w(\rho, \boldsymbol{\theta})=\nabla_{\boldsymbol{\theta}} V(\rho, \boldsymbol{\theta})
$$

on $\rho=\rho(\boldsymbol{\theta}), \boldsymbol{\theta} \in \Omega_{0}$. Moreover, by (5.4)

$$
\frac{\partial w}{\partial \rho}=-\frac{2}{(p-1)} \frac{w}{\rho}=-\frac{2 V}{(p-1) \rho}=\frac{\partial V}{\partial \rho}
$$

on $\rho=\rho(\boldsymbol{\theta}), \boldsymbol{\theta} \in \Omega_{0}$. Therefore, on the $C^{1}$ manifold given by $\rho=\rho(\boldsymbol{\theta}), w$ and $\nabla w$ agree with $V$ and $\nabla V$ and therefore, by unique continuation,

$$
w(r, \theta)=V(r, \boldsymbol{\theta})
$$

on $(0, \infty) \times \Omega_{0}$. Hence $w$ is singular.

We next prove the following:

Theorem 5.2. If $G(\rho, \boldsymbol{\theta})$ given by (5.4) changes sign on $(0, \infty) \times \Omega$, then $w$ cannot be a positive solution of (4.1) satisfying the boundary condition.

Proof. The hypothesis assures us that there is an open subset $\Omega_{1} \subset \Omega$ such that for each $\boldsymbol{\theta} \in \Omega_{1}$, there is an interval $I_{\boldsymbol{\theta}} \subset(0, \infty)$ with $G(\rho, \boldsymbol{\theta})<0$ if $\rho \in I_{\theta}$. On the other hand, from the mean value theorem, for each such $\theta$ and each $\delta>0$, there is a $\rho_{+}(\boldsymbol{\theta})$ with $0<\rho_{+}(\boldsymbol{\theta})<\delta$ such that $G\left(\rho_{+}(\boldsymbol{\theta}), \boldsymbol{\theta}\right)>0$. Therefore $I_{\theta}$ cannot have zero as its right-hand end point. 
It follows from these observations and the continuity of $G$ that there is an open set $\Omega_{0} \subset \Omega_{1}$, a number $a>0$ such that $G(a, \theta)$, and a number $b>a$ such that $G(b, \boldsymbol{\theta})<0$ for all $\boldsymbol{\theta} \in \bar{\Omega}_{0}$. Therefore the number

$$
\rho_{0}(\boldsymbol{\theta})=\inf \{\rho \mid G(\rho, \boldsymbol{\theta}) \leq 0, \rho \in[a, b]\}
$$

is well defined, and $G\left(\rho_{0}(\boldsymbol{\theta}), \boldsymbol{\theta}\right)=0$ for $\boldsymbol{\theta} \in \bar{\Omega}_{0}$. Moreover $G(\rho, \boldsymbol{\theta})>0$ if $a \leq \rho<\rho_{0}(\boldsymbol{\theta})$ and $\boldsymbol{\theta} \in \bar{\Omega}_{0}$.

Let

$$
S=\left\{\left(\rho_{0}(\boldsymbol{\theta}), \boldsymbol{\theta}\right) \mid \boldsymbol{\theta} \in \overline{\boldsymbol{\Omega}}_{0}\right\}
$$

denote the graph of the function $\rho_{0}$. Then $S \subset[a, b] \times \bar{\Omega}_{0}$, and, from Lemma 1.8 of [11],

$$
H^{N-1}(S) \geq H^{N-1}\left(\bar{\Omega}_{0}\right)>0,
$$

where $H^{k}(S)$ denotes the $k$-dimensional Hausdorff measure of $(S)$.

Let

$$
T=\left\{\left(\rho_{0}(\boldsymbol{\theta}), \boldsymbol{\theta}\right) \in S \mid \nabla G(\rho(\boldsymbol{\theta}), \boldsymbol{\theta})=\mathbf{0}\right\} .
$$

We will show that $T$ has $N-1$ dimensional Hausdorff measure zero or else $w$ is singular. Suppose the former. Then there are points in $S$ where $\nabla G \neq 0$. Let $\left(\rho_{0}\left(\boldsymbol{\theta}_{0}\right), \boldsymbol{\theta}_{0}\right)$ be such a point. If $G_{\rho}\left(\rho_{0}\left(\boldsymbol{\theta}_{0}\right), \boldsymbol{\theta}_{0}\right) \neq 0$, then in a neighborhood of this point, the implicit function theorem assures us that we may write $\rho=$ $\rho_{1}(\boldsymbol{\theta})$ with $\rho_{1} \in C^{1}\left(\widetilde{\Omega}_{0}\right), G\left(\rho_{1}(\boldsymbol{\theta}), \boldsymbol{\theta}\right)=0$, for all $\boldsymbol{\theta}$ in some open set $\widetilde{\Omega}_{0} \subset$ $\Omega_{0}$ and $\rho_{1}\left(\boldsymbol{\theta}_{0}\right)=\rho\left(\boldsymbol{\theta}_{0}\right)$. In this case the result follows from the previous lemma. If $\nabla_{\boldsymbol{\theta}} G\left(\rho_{0}\left(\boldsymbol{\theta}_{0}\right), \boldsymbol{\theta}_{0}\right) \neq \mathbf{0}$, then one of the angular variables, say $\theta_{1}$, may be expressed as a $C^{1}$ function of $\rho$ and $\theta_{2}, \ldots, \theta_{N-1}$. That is, $\theta_{1}=$ $\Theta\left(\rho, \theta_{2}, \ldots, \theta_{N-1}\right)$ and $\theta_{10}=\Theta\left(\rho_{0}\left(\boldsymbol{\theta}_{0}\right), \theta_{20}, \ldots, \theta_{(N-1) 0}\right)$ where $\Theta$ is a $C^{1}$ function of $\left(\rho, \theta_{2}, \ldots, \theta_{N-1}\right)$ is an open neighborhood of $\left(\rho_{0}\left(\boldsymbol{\theta}_{0}\right), \theta_{20}, \ldots\right.$, $\left.\theta_{(N-1) 0}\right)$. Moreover, $G\left(\rho, \Theta\left(\rho, \theta_{2}, \ldots, \theta_{N-1}\right), \theta_{2}, \ldots, \theta_{N-1}\right)=0$ on this set. If $\partial \Theta / \partial \rho \neq 0$ at some point of this neighborhood, we may again apply the implicit function theorem to find $\rho$ as a $C^{1}$ function of $\boldsymbol{\theta}$ on some $\widetilde{\Omega}_{0} \subset \Omega$ and apply and the lemma again. If $\partial \Theta / \partial \rho \equiv 0$ then

$$
G\left(\rho, \Theta\left(\theta_{2}, \ldots, \theta_{N-1}\right), \theta_{2}, \ldots, \theta_{N-1}\right) \equiv 0
$$

for $\theta_{i}=\theta_{i 0}$ and all $\rho$ in some small open neighborhood of $\rho_{0} \equiv \rho\left(\boldsymbol{\theta}_{0}\right)$. However, by construction, $G$ cannot vanish for $a<\rho<\rho_{0}$ and $\boldsymbol{\theta}=\boldsymbol{\theta}_{0}$. This is the desired contradiction.

In order to show that $H^{N-1}(T)=0$, we let

$$
l(r, \boldsymbol{\theta})=r^{2 /(p-1)} w(r, \boldsymbol{\theta}) .
$$

Then

$$
G(r, \boldsymbol{\theta})=r^{(p-3) /(p-1)} l_{r}(r, \boldsymbol{\theta}) .
$$

A routine calculation shows that

$$
r^{2} l_{r r}+(N-1-4 /(p-1)) r l_{r}+\nu l+\Delta_{\theta} l+l^{p}=0
$$


where $\nu=2 /(p-1)(2 /(p-1)+2-N)$ as before. If we differentiate both sides of (5.8) with respect to $r$, we see that on $[a, b] \times \bar{\Omega}_{0}$, there are constants $A$, $B$ such that

$$
|\Delta G| \leq A|G|+B|\nabla G|,
$$

an inequality considered by Caffarelli and Friedman [1]. From this article we conclude that $H^{N-1}(T)=0$ unless $G \equiv 0$ on $[a, b] \times \bar{\Omega}_{0}$. In the latter case, it follows that $w=r^{-2 /(p-1)} F(\theta)$ on this set, where $F$ solves (3.3), and consequently (by unique continuation again) $w=r^{-2 /(p-1)} F(\boldsymbol{\theta})$ on $(0, \infty) \times \bar{\Omega}_{0}$ and hence is singular. Thus, the theorem is established.

Remark 5.1. The unique continuation results of $[21,23]$ can also be applied here.

\section{NONEXISTENCE OF STATIONARY SOLUTIONS UNDER SINGULAR SOLUTIONS}

Let $p>1-2 / \gamma_{-}$. Then there exist singular stationary solutions $u_{s}=$ $r^{-2 /(p-1)} \alpha(\theta)$ as constructed in $\S 3$. In this section we show that regular positive stationary solution $w$, when they exist, cannot satisfy $w \leq u_{s}$ for some $u_{s}$, provided $p$ is not too large.

Theorem 6.1. Under the condition

$$
1-2 / \gamma_{-}<p \leq(N+2) /(N-2)
$$

no regular solution of (4.1) satisfying $w \leq u_{s}$ can exist except $w \equiv 0$.

Proof. Let $w$ be such a positive stationary solution. By the results of $\S 5$, we may assume that $G(r, \theta) \geq 0$ on $D$. Therefore, we may assume that since

$$
l(r, \boldsymbol{\theta}) \equiv r^{2 /(p-1)} w(r, \boldsymbol{\theta}) \leq \alpha(\boldsymbol{\theta}),
$$

and

$$
l_{r}=\left(r^{2 /(p-1)} w\right)_{r} \geq 0
$$

we must have

$$
\int_{\Omega} l_{r}(r, \boldsymbol{\theta}) \alpha(\boldsymbol{\theta}) d S_{\boldsymbol{\theta}}>0
$$

for all $r>0$. (If the integrand vanished for some $r_{0}>0$ on an open set $\Omega_{0} \subset \Omega$, we could find a $C^{1}$ solution of (5.4) and be finished by the results of $\S 5$.

Therefore, if we define

$$
L(r)=\int_{\Omega} l(r, \boldsymbol{\theta}) \alpha(\boldsymbol{\theta}) d \boldsymbol{\theta},
$$

we find from (5.8) that with $q \equiv 2 /(p-1)$

$$
0=L_{r r}+(N-1-2 q) L_{r} / r+\int_{\Omega} l \alpha\left(l^{p-1}-\alpha^{p-1}\right) d S_{\theta} / r^{2} .
$$


The integral on the right of (6.4) is nonpositive by (6.2). Consequently we find for $r>r_{1}$

$$
L_{r}(r) \geq\left(r / r_{1}\right)^{2 q+1-N} L_{r}\left(r_{1}\right),
$$

and, after a second quadrature, for some $c>0$

$$
L(r) \geq L\left(r_{1}\right)+c \cdot \begin{cases}r^{2 q+2-N}, & p<(N+2) /(N-2), \\ \ln r, & p=(N+2) /(N-2) .\end{cases}
$$

Therefore, $L(r)$ is unbounded as $r \rightarrow \infty$. However, by (6.2) again

$$
L(r) \leq \int_{\Omega} \alpha^{2}(\boldsymbol{\theta}) d S_{\boldsymbol{\theta}},
$$

an obvious contradiction. Thus $l_{r}$ and hence $G$ must change sign.

Remark 6.1. Since for $N \geq 3$

$$
(N+2) /(N-2)<(N+1) /(N-3),
$$

we have not eliminated the possibility of stationary solutions under singular solutions for all $p$ for which singular solutions exist.

\section{Global eXistence}

Suppose that

$$
1-2 / \gamma_{-}<p< \begin{cases}(N+1) /(N-3), & N>3, \\ \infty, & N=2,3 .\end{cases}
$$

Then singular stationary solutions exist. Let $u_{s}$ be one such of the form $r^{-2 /(p-1)} \alpha(\theta)$. We show that if

$$
0 \leq u_{0} \leq \min \left\{r^{\varepsilon}, u_{s}\right\}
$$

for some $\varepsilon>0$, then $u\left(x, t ; u_{0}\right)$ is global. If

$$
1-2 / \gamma_{-}<p \leq(N+2) /(N-2),
$$

then

$$
\lim _{t \rightarrow \infty} u\left(x, t ; u_{0}\right)=0
$$

Let

$$
u_{s}=\alpha(\boldsymbol{\theta}) r^{-2 /(p-1)}
$$

and define

$$
\psi(r, \boldsymbol{\theta})=A r^{m} \alpha(\boldsymbol{\theta}),
$$

where, for $\nu$ as defined in $\S 3, m$ satisfies

$$
-\frac{1}{2}(N-2)-\sqrt{\nu+\left(\frac{N-2}{2}\right)^{2}}<m<-\frac{1}{2}(N-2)+\sqrt{\nu+\left(\frac{N-2}{2}\right)^{2}}
$$


and $A>0$. Then

$$
\begin{aligned}
\Delta \psi+\psi^{p}= & A \alpha(\boldsymbol{\theta}) r^{(m-2)}[m(m+N-2)-\nu] \\
& -A r^{(m-2)} \alpha^{p}(\boldsymbol{\theta})\left[1-A^{p-1} r^{m(p-1)+2}\right] .
\end{aligned}
$$

Then

$$
\Delta \psi+\psi^{p} \leq 0
$$

in

$$
D_{A}=D \cap\left\{r \leq A^{-[(p-1) /(m(p-1)+2)]}\right\} .
$$

Let

$$
\bar{u}(r, \boldsymbol{\theta})=\inf \left\{u_{s}, \psi\right\}= \begin{cases}\psi & \text { in } D_{A}, \\ u_{s} & \text { in } D-D_{A} .\end{cases}
$$

Then $u$ is a supersolution in the weak sense. Therefore, by standard arguments, the solution of $(1.1), u(x, t, \bar{u})$, with $u_{0}=u(x, 0 ; \bar{u})=\bar{u}$ exists globally.

Lemma 7.1. $u(x, t, \bar{u})$ is decreasing in $t$.

Proof. This follows from a result of [13].

\section{Lemma 7.2.}

$$
\lim _{t \rightarrow+\infty} u(x, t ; \bar{u})=w(x)
$$

where $w(x)$ is a stationary solution of $(1.1)$

Proof. See Sattinger [12].

Lemma 7.3. If (7.3) holds, then $w(x) \equiv 0$.

This follows from the preceding section.

Theorem 7.4. If (7.1) holds and $u_{0}$ satisfies (7.2), then $u\left(x, t ; u_{0}\right) \leq u(x, t, \bar{u})$ and (by standard arguments) is global. If (7.2) and (7.3) hold, then (7.4) holds also.

Remark 7.5. If we combine our result with that of Fujita, we see that if

$$
p>\min \left(1+2 / N, 1-2 / \gamma_{-}\right),
$$

global solutions of (1.1) exist for certain nontrivial initial values.

8. BLOWUP IN EXTERIOR DOMAINS

In this section we indicate how some of the arguments in $\S 2$ carry over to other unbounded regions, specifically regions exterior to bounded regions.

Lemma 8.1. Let $D_{R}=\left\{x \in R^{N}|| x \mid>R\right\}$. Then there is not positive regular global solution of

$$
\begin{aligned}
u_{t} & =\Delta u+u^{p} & & \text { in } D_{R} \times(0, \infty), \\
u(x, 0) & =u_{0}(x) & & \text { in } D_{R}, \\
u(x, t) & =0 & & \text { on } \partial D_{R} \times(0, \infty),
\end{aligned}
$$


when

$$
1<p<1+2 / N \text {. }
$$

Proof. We only sketch the proof. Let $m \in(0,2 /(p-1)-N)$ and let $\rho \gg R$ be such that

$$
1-m \ln (\rho / R)<0 \text {. }
$$

Let

$$
\varphi(r)= \begin{cases}r^{m} e^{-k r}, & r>\rho, \\ A \ln (r / R), & R<r<\rho .\end{cases}
$$

We choose $A$ such that $\varphi(\rho+)=\varphi(\rho-)$ and $k>0$ but so small that $\varphi^{\prime}(\rho+) \geq$ $\varphi^{\prime}(\rho-)$ and let

$$
\tilde{u}(r, t):=\omega_{N}^{-1} \int_{S^{N-1}} u(x, t) d S_{\theta}
$$

where $\omega_{N}=$ area of $S^{N-1}$.

Then with

$$
G(t):=C^{-1} \int_{R}^{\infty} \varphi(r) \tilde{u}(r, t) r^{N-1} d r
$$

where

$$
C:=\int_{R}^{\infty} \varphi(r) r^{N-1} d r=O\left(k^{-(m+M)}\right) \quad(k \rightarrow 0),
$$

we have $G^{\prime} \geq-\lambda G+G^{p}$ and $G(0)>\lambda^{1 /(p-1)}$ when we take

$$
\lambda=k^{2}\left(m+\frac{1}{2}(N-1)\right)^{2} / m(m+N-2)
$$

and $k$ sufficiently small. We can again apply Lemma 2.2 to obtain the result.

Theorem 8.1. Let $D \subset R^{N}$ and let $D^{C}=R^{N}-D$ be bounded. If (8.2) holds, there are no quasiregular global solutions of (8.1).

Proof. The result will follow from (8.1) by comparison. Let $u_{0}>0$ on some open subset of $D$ and let $0<u_{1}<u_{0}$ have compact support. Let

$$
v_{i}(x, t)=u\left(x, t ; u_{i}\right), \quad i=0,1 .
$$

Then $v_{i}$ exists on $D \times\left(0, T_{i}\right)$ where $T=T_{0} \leq T_{1}$ and

$$
v_{1}(x, t) \leq v_{0}(x, t) \text { on } \bar{D} \times\left[0, T_{0}\right) .
$$

Now let $R>0$ be so large that $D_{R} \subset D$ and let $v$ be a regular solution of (8.1) on $D_{R} \times\left[0, T_{2}\right)$ where

$$
v(x, 0)=v_{1}\left(x, \frac{1}{2} T_{0}\right), \quad x \in D_{R} .
$$

Then, by comparison,

$$
v(x, t) \leq v_{1}(x, t) \leq v_{0}(x, t)=u(x, t)
$$

while $T_{0} \leq T_{1} \leq T_{2}$. However, by Lemma 8.2, $T_{2}<\infty$. Therefore $T_{0}<\infty$ and we are done. 
Remark 8.2. Let $N>2$. It is easy to see that if $p>N /(N-2)>1+2 / N$, then

$$
u_{s}(r, \boldsymbol{\theta})=\lambda r^{-2 /(p-1)}
$$

solves $\Delta u+u^{p}=0$ in $R^{N}-\{0\}$ where

$$
\lambda \equiv\left[\frac{2}{p-1}\left(N-2-\frac{2}{p-1}\right)\right]^{1 /(p-1)} .
$$

Theorem 8.3. Let $D^{C}=R^{N}-D$ be a bounded region of $R^{N}$ and let $u_{0} \in C(\bar{D})$ $\left(u_{0} \geq 0\right)$.

(i) If $p>1+2 / N$ then there are nontrivial global solutions of (8.1).

(ii) If $N /(N-2)<p<(N+2) /(N-2)$, then $u$ decays to zero pointwise at $t \rightarrow \infty$.

(iii) If $N \geq 3, p>N /(N-2)$, and $u_{0} \leq u_{s}$, then the corresponding solution of $(8.1)$ is global.

The first statement follows by comparison with a Fujita solution. To prove (ii) we let $R>0$ be such that $D \subset D_{R}$ and we use $u_{s}$ as a super solution of $(8.1)$ in $\bar{D}_{R} \times[0, \infty)$. The third statement follows by an argument used to establish an analog of Theorem 6.1.

Remark 8.2. Combining Theorem 8.1 and the first statement of Theorem 8.3, we obtain the classical Fujita result for any domain with bounded complement.

\section{CONCLUDING REMARKS}

During the course of preparation of this paper, we became aware of reference [14], where stationary solutions of

$$
\Delta w+|x|^{\sigma} w^{p}=0, \quad x \in R^{N}, p>1, \sigma \in R,
$$

are discussed.

The results of [14] lead us to consider the problem (with $\sigma>-2$ )

$$
\begin{aligned}
\partial u / \partial t & =\Delta u+|x|^{\sigma} u^{p} & & \text { in } D \times(0, T), \\
u & =0 & & \text { on } \partial D \times(0, T), \\
u(x, 0) & =u_{0}(x) & & \text { on } D,
\end{aligned}
$$

where $u_{0} \geq 0$. We shall state, but not prove, results for $(9.1)$ which are analogous to Theorem 2.3, Theorem 3.1, Theorem 4.6, Theorem 5.2, and Theorem 7.4 .

With the exception of Theorem 2.3, the results are obtained at easy modifications of the proofs already given. We have:

Theorem 9.1. Let $u$ be a regular solution of $(9.1)$ with $u_{0} \geq 0, u_{0} \not \equiv 0$. Let $\gamma_{ \pm}$ be as in $\S 1$.

(i) If

$$
1<p<1+(2+\sigma) /\left(2-\gamma_{-}\right)
$$

then $u$ cannot be global. 
(ii) If

$$
1<p<1+(2+\sigma) /\left(-\gamma_{-}\right)
$$

then (9.1) cannot have positive stationary solutions in $D$.

(iii) If

$$
1 \leq 1+(2+\sigma) /\left(-\gamma_{-}\right)<p< \begin{cases}(N+1) /(N-3) & \text { if } N \geq 4, \\ \text { arbitrary } & \text { if } N=2,3,\end{cases}
$$

then there exist singular stationary solutions (9.1) of the form

$$
u_{s}(\boldsymbol{\theta})=r^{-(2+\sigma) /(p-1)} \alpha(\boldsymbol{\theta}) .
$$

If $1<p \leq 1+(2+\sigma) /\left(-\gamma_{-}\right)$, no such singular solutions exist.

(iv) If

$$
1 \leq 1+(2+\sigma) /\left(-\gamma_{-}\right)<p<\min \left\{\frac{N+1}{N-3}, \frac{N+2+2 \sigma}{N-2}, \infty\right\}
$$

then regular solutions of $(9.1)$ with $u(x, 0) \leq u_{s}(x)$ are global and decay to zero pointwise as $t \rightarrow+\infty$.

(v) If

(9.6) $1 \leq 1+\frac{2+\sigma}{\left(-\gamma_{-}\right)}<\frac{N+2+2 \sigma}{N-2}<p< \begin{cases}(N+1) /(N-3), & N \geq 4, \\ \infty, & N=3,\end{cases}$

then regular solutions of $(9.1)$ with $u(x, 0) \leq u_{s}(x)$ are global.

The proof of the first statement follows the lines of that of Theorem 2.3 if we first make the change of variables

$$
v=r^{\delta} u
$$

in (9.1) $(r=|x|)$ where $\delta=\sigma /(p-1)$. Then

$$
\begin{aligned}
v_{t}= & r^{-(N-1-2 \delta)} \frac{\partial}{\partial r}\left(r^{(N-1-2 \delta)} \frac{\partial v}{\partial r}\right) \\
& +r^{-2}\left(\Delta_{\theta} v-\delta(N-2-\delta) v\right)+v^{p} .
\end{aligned}
$$

Then, with $\tilde{v}$ replacing $\tilde{u}$ and

$$
G(t):=\int_{0}^{\infty} \tilde{v} \Phi_{0} r^{N-1} d r
$$

we see that (2.3) will hold provided (2.2) holds for all $r$ with $m$ replaced by $m+\delta$. Thus, $m$ must satisfy

$$
\gamma_{+}-\delta<m<2(p-1)-N
$$

which leads to condition (9.2). When $\omega_{1} \rightarrow 0$, we obtain

$$
1<p<1+(2+\sigma) / N=(N+2+\sigma) / N .
$$


The proofs of (ii)-(iv) are easy modifications of the arguments of $\S \S 3-7$. When $\omega_{1} \rightarrow 0$ in (9.3) we see that there can be no stationary solutions if

$$
1<p<(N+\sigma) /(N-2)
$$

so that we recover Theorem A.1 of [14].

\section{NOTES ADDED LATER}

1. Recently, using comparison arguments of Meier [10] but avoiding the use of the Green's function, Levine and Meier [16] proved for (9.1) and hence for (1.1) when $\sigma=0$ that there are nontrivial nonnegative almost regular solutions of (9.1) if $\sigma>0$ and

$$
p>1+(2+\sigma) /\left(2-\gamma_{-}\right) .
$$

Thus when $\sigma \geq 0, p(\sigma) \equiv 1+(2+\sigma) /\left(2-\gamma_{-}\right)$is the cutoff point between the blowup case and the global existence case (for small data). They did not prove that $p(\sigma)$ belongs to the blowup case.

2. At about the time this paper was accepted for publication, one of us (Bandle) learned of some related work of Kavian [17] and others [18, 19]. There results were obtained quite independently from ours and the proofs are much different. They are carried out in the context of $L^{2}$ theory.

At the risk of doing an injustice to these workers, let us briefly describe their results. Let

$$
K(y)=\exp \left(\frac{1}{4}|y|^{2}\right), \quad y \in D .
$$

They introduce the Hilbert spaces

$$
\begin{aligned}
L^{2}(K, D) & =\left\{\left.f\left|\int_{D}\right| f\right|^{2} K d x<\infty\right\}, \\
H_{0}^{1}(K, D) & =\left\{f \mid f, \nabla f \in L^{2}(K, D) \text { and } f=0 \text { on } \partial D\right\} .
\end{aligned}
$$

They require solutions of $(1.1)$ to belong, at each $t$, to $H_{0}^{1}(K, D)$ or to $H_{0}^{1}(D)$. They define for $f \in H_{0}^{1}(D)$

$$
L f=-\Delta f(\mathbf{x})-\frac{1}{2} \mathbf{x} \cdot \nabla f(\mathbf{x}) \quad(\mathbf{x} \in D)
$$

and denote by $\lambda_{1}$ the smallest eigenvalue of $L$. They assume throughout that $D$ is convex and $1<p<(N+2) /(N-2)$. They prove the following (for maximal solutions):

(a) If $u(\cdot) \in H_{0}^{1}(D), u_{0} \geq 0$, and $1<p \leq 1+1 / \lambda_{1}$, then $u(t)$ blows up in finite time.

(b) If $p \geq 1+1 / \lambda_{1}, p<(N+2) /(N-2)$,

$$
\begin{aligned}
E(0) \equiv & \frac{1}{2} \int_{D}|u(0)|^{2} K d x-\frac{1}{p+1} \int_{\Omega}|u(0)|^{p+1} K d y \\
& -\frac{1}{2(p-1)} \int_{\omega}|u(0)|^{2} K d y \leq 0,
\end{aligned}
$$

then $u$ blows up in finite time. 
(c) If $p>1+1 / \lambda_{1}$ and $p<(N+2) /(N-2)$ then there are global solutions for some nontrivial $u(0) \in H_{0}^{1}(K<D)$.

(d) If $p>1+1 / \lambda_{2}, p>(N+2) /(N-2)$, and $u(t)$ is a global solution, then $u(t)$ is global and the $C^{2}(\Omega)$ norm decays at least as fast as $t^{-1 /(p-1)}$ as $t \rightarrow+\infty$.

They rely on potential well arguments of Payne and Sattinger [20] for the global existence and on the concavity arguments of [8] as modified by Payne and Sattinger [20] for the global nonexistence. The Hilbert space approach necessitates the additional restriction that

$$
p<(N+2) /(N-2),
$$

which neither we nor Levine and Meier require. On the other hand, they prove that $1+1 / \lambda_{1}$ does belong to the blowup case. For cones, an elementary argument show that

$$
\lambda_{1}=\frac{1}{2}\left(2-\gamma_{-}\right)=\frac{1}{2}\left(N+\gamma_{+}\right) .
$$

They do not consider the case of global existence when $p \geq(N+2) /(N-2)$ nor do they consider the case of (9.1).

It should also be noted that the authors of [17-19] work in $H_{0}^{1}(D)$, whereas we work in the space

$$
\left\{f \mid \forall k>0, \int_{D} e^{-k|\mathbf{x}|}[|f(\mathbf{x})|+|\nabla f(\mathbf{x})|] d x<\infty \text { and } f=0 \text { on } \partial D\right\},
$$

which strictly contains $H_{0}^{1}(D)$.

However, the decay result (d), which overlaps ours, contains more information as the decay rate is precisely specified. Meier and Levine obtain a somewhat better decay rate for the $L^{\infty}$ norm under certain conditions. They show that

$$
\limsup _{t \rightarrow+\infty}\left(t+t_{0}\right)^{(1 / 2)\left(N+\gamma_{+}\right)}\|u(t)\|_{\infty}<\infty
$$

for solutions which initially lie under the supersolutions they construct, for all $p>1+2 /\left(N+\gamma_{+}\right)$. This is clearly a better estimate of the rate of decay than (d) for the $L^{\infty}$ norm.

It would be of interest to know if the result of (d) is optimal. That is, is it true that there are solutions which decay exactly like $t^{-1 /(p-1)}$, that is, that

$$
\lim _{t \rightarrow+\infty} t^{1 /(p-1)}\|u(t)\|_{L^{\infty}}
$$

exists and is nonzero?

Acknowledgments. The authors thank Matts Essen for his suggestions leading to the results of $\S 4$, Gary Lieberman for bringing [1] to their attention, Paul Sacks and Brian Straughan for several useful comments including the application of the ideas of earlier sections to $\S 8$, and Steve Willson for bringing [11] to their attention. 


\section{REFERENCES}

1. L. A. Caffarelli and A. Friedman, Partial regularity of the zero set of solutions of linear and superlinear elliptic equations, J. Differential Equations 60 (1985), 420-433.

2. H. Fujita, On the blowing up of solutions of the Cauchy problem for $u_{t}=\Delta u+u^{1+\alpha}$, J. Fac. Sci. Tokyo Sect. IA Math. 13 (1966), 102-124.

3. _ On some nonexistence and nonuniqueness theorems for nonlinear parabolic equations, Proc. Sympos. Pure Math., vol. 18, Amer. Math. Soc., Providence, R.I., 1969, pp. 105-113.

4. A. Friedman, Partial differential equations of parabolic type, Prentice-Hall, Engelwood Cliffs, N.J., 1964.

5. P. Grisvard, Elliptic problems in nonsmooth domains, Pitman, London, 1985.

6. S. Kaplan, On the growth of quasilinear parabolic equations, Comm. Pure Appl. Math. 16 (1963), 305-330.

7. V. A. Kondrat'ev and O. A. Olenik, Boundary value problems for partial differential equations in non-smooth domains, translated in Russian Math. Surveys 38 (2) (1983), 1-86.

8. H. A. Levine, Some nonexistence and instability theorems for solutions of formally parabolic equations of the form $P u_{t}=-A u+F(u)$, Arch. Rational Mech. Anal. 51 (1973), 371-286.

9. P. Meier, Existence et non-existence de solutions globales d'une équation de la chaleur semilinéaire: extension d'un théorème de Fujita, C. R. Acad. Sci. Paris Sér. I 303 (1986), 635-637.

10. $\ldots$ Blow up of solutions of semilinear parabolic differential equations, $\mathrm{Z}$. Angew. Math. Phys. 39 (1988) 135-149.

11. K. J. Falconer, The geometry of fractals sets, Cambridge University Press, New York, 1985.

12. D. H. Sattinger, Topics in stability and bifurcation theory, Lecture Notes in Math., vol. 309, Springer, New York, 1973.

13. _. Monotone methods in nonlinear elliptic and parabolic boundary value problems, Indiana Univ. Math. J. 21 (1972), 979-1000.

14. B. Gidas and J. Spruck, Global and local behavior of positive solutions of nonlinear elliptic equations, Comm. Pure Appl. Math. 23 (1981), 525-598.

15. W.-M. Ni, A nonlinear Dirichlet problem on the unit ball and its applications, Indiana Univ. Math. J. 31 (1982), 289-304.

16. H. A. Levine and P. Meier, The value of the critical exponent for reaction-diffusion equations in arbitrary cones (to appear).

17. O. Kavian, Remarks on the large-time behavior of a nonlinear diffusion equation, Ann. Inst. H. Poincaré-Analyse Nonlinéaire 4 (1987), 423-452.

18. M. Escobedo and O. Kavian, Asymptotic behavior of positive solutions of a nonlinear heat equation (preprint).

19. __ Variational problems related to self similar solutions of the heat equations, J. Nonlinear Anal. TMA 11 (1987), 1103-1133.

20. L. E. Payne and D. H. Sattinger, Saddle points and instability of nonlinear hyperbolic equations, Israel J. Math. 22 (1975), 273-303.

21. R. Hardt and L. Simon, Nodal sets for solutions of elliptic equations, Centre for Math. Anal., Report CMA-R25-87, Australian Nat. Univ., Canberra, Australia, 1987.

22. Wi-Ming Ni and J. Serrin, Existence and nonexistence theorems for ground states of quasilinear partial differential equations. The anomalous case, Acad. Naz. Lincei 77 (1986), 231-257.

23. Luc Robbiano, Sur les zeros de solutions d'inequalities differentielles elliptiques, Comm. Partial Differential Equations 72 (1987), 903-919.

Mathematisches Institute der Universität Basel, Basel, Switzerland CH-4051

Department of Mathematics, Iowa State University, Ames, Iowa 50011 